\title{
Textbook Gerhard K. Lang: Ophthalmology, 3rd Edition, 384 pages, 600 pictures, published 2015 ISBN: 978-3-13-126163-2 Thieme Company, New York
}

\author{
Bernd Kirchhof ${ }^{1}$
}

Received: 2 March 2016 / Accepted: 28 March 2016/Published online: 6 April 2016

(C) Springer-Verlag Berlin Heidelberg 2016

This is a well-established and recognized textbook of the whole ophthalmology for medical students.

The strength of this book is its many pictures of typical disease manifestations, supplemented by schematic drawings, a "picture book" as the author points out. The combination of pictures and drawings greatly helps learning, memorization, and understanding of pathogenesis and typical pathologies. Yet current knowledge of the pathogenesis of diabetic retinopathy with an emphasis on inflammatory mechanisms (leucocyte adhesion) is missing.

Highlighted mnemonics help to emphasize key messages, and the list of cardinal symptoms facilitates the structuring of a huge body of information. The overall emphasis of the textbook is on disease recognition.

The flaws of this textbook are on the side of therapy:

For example, current progress in the treatment of neovascular age-related macular degeneration (nvAMD) is not sufficiently accounted for. After all, science magazines highlighted VEGF blockers for nvAMD in 2006 as the most important progress in medicine. Spectral domain OCT imaging is of equally major significance. Detailed knowledge of SD-OCT interpretation is essential for indication of timely treatment and re-treatment with VEGF blockers. Laser treatment and photodynamic therapy are listed but no longer merit significant consideration.

Bionics are being introduced into routine treatment now. Therefore, examples such as the artificial retina (retinal implant) should have been listed for treatment of end-stage retinitis pigmentosa.

New treatments for corneal diseases (Descemet's stripping endothelial keratoplasty, penetrating keratoplasty) merit more space.

Lang's Ophthalmology is a well-established label amongst medical students in and outside Germany. It is ambitious to attempt to cover all of ophthalmology in 384 pages. But the claim for systematic coverage with excellent graphical material must include recent and significant advances in therapy. The hope for the next edition is in the new and younger co-authors. Their input and responsibility merit visibility on the cover page.

Bernd Kirchhof

bekirchhof@googlemail.com

1 Center of Ophthalmology, University of Koeln, Koeln, Germany 